\title{
Non-linear Functional Brain Co-activations in Short-Term Memory Distortion Tasks
}

\author{
Anna Ceglarek ${ }^{1 *}$, Jeremi K. Ochab², Ignacio Cifre ${ }^{3}$, Magdalena Fafrowicz', \\ Barbara Sikora-Wachowicz'1, Koryna Lewandowska' ${ }^{1}$, Bartosz Bohaterewicz'1, \\ Tadeusz Marek ${ }^{1}$ and Dante R. Chialvo ${ }^{4,5}$ \\ ${ }^{1}$ Department of Cognitive Neuroscience and Neuroergonomics, Institute of Applied Psychology, Jagiellonian University, \\ Krakow, Poland, ${ }^{2}$ M. Kac Complex Systems Research Center and M. Smoluchowski Institute of Physics, Jagiellonian \\ University, Krakow, Poland, ${ }^{3}$ Facultat de Psicologia, Ciències l'Educació i de l'Esport, Blanquerna, Universitat Ramon Llull, \\ Barcelona, Spain, ${ }^{4}$ Center for Complex Systems and Brain Sciences (CEMSC3), Instituto de Ciencias Físicas (ICIFI), Escuela \\ de Ciencia y Tecnología, Universidad Nacional de San Martín (UNSAM), Buenos Aires, Argentina, ${ }^{5}$ Consejo Nacional \\ de Investigaciones Científicas y Técnicas (CONICET), Buenos Aires, Argentina
}

\section{OPEN ACCESS}

Edited by:

Gopikrishna Deshpande, Auburn University, United States

Reviewed by:

Xiaowei Zhuang,

Lou Ruvo Center for Brain Health,

Cleveland Clinic, United States

Guorong $W u$,

Ghent University, Belgium

${ }^{*}$ Correspondence: Anna Ceglarek

anna.ceglarek@uj.edu.pl

Specialty section:

This article was submitted to Brain Imaging Methods,

a section of the journal

Frontiers in Neuroscience

Received: 16 September 2021 Accepted: 04 November 2021 Published: 03 December 2021

Citation:

Ceglarek A, Ochab JK, Cifre I, Fafrowicz M, Sikora-Wachowicz B, Lewandowska K, Bohaterewicz B,

Marek T and Chialvo DR (2021)

Non-linear Functional Brain

Co-activations in Short-Term Memory

Distortion Tasks.

Front. Neurosci. 15:778242.

doi: 10.3389/fnins.2021.778242
Recent works shed light on the neural correlates of true and false recognition and the influence of time of day on cognitive performance. The current study aimed to investigate the modulation of the false memory formation by the time of day using a non-linear correlation analysis originally designed for $\mathrm{fMRI}$ resting-state data. Fifty-four young and healthy participants (32 females, mean age: $24.17 \pm 3.56$ y.o.) performed in MR scanner the modified Deese-Roediger-McDermott paradigm in short-term memory during one session in the morning and another in the evening. Subjects' responses were modeled with a general linear model, which includes as a predictor the nonlinear correlations of regional BOLD activity with the stimuli, separately for encoding and retrieval phases. The results show the dependence of the non-linear correlations measures with the time of day and the type of the probe. In addition, the results indicate differences in the correlations measures with hippocampal regions between positive and lure probes. Besides confirming previous results on the influence of time-of-day on cognitive performance, the study demonstrates the effectiveness of the non-linear correlation analysis method for the characterization of fMRI task paradigms.

Keywords: fMRI, DRM paradigm, memory distortions, short-term (working) memory, non-linear correlations, point process analysis, functional dynamic connectivity

\section{INTRODUCTION}

The process of cognitive control supports adaptive responses and inhibits automatic ones. It is linked to the working memory not only by function but also by location in the brain-in the prefrontal cortex (for review, see: Miller, 2000). Cognitive control is also enormously involved in decision-making processes to obtain appropriate responses to changing environmental conditions. A model of simple, two-choice decisions that has gained popularity in recent years is the driftdiffusion model (DDM; Ratcliff, 1978; Ratcliff and McKoon, 2008). It describes the decision-making process as an accumulation of evidence about a stimulus from perceptual organs or memory, leading to a reaction (most often a motor one) when the evidence exceeds a certain threshold. The studies investigating neural underpinnings of decision-making focus mainly on the prefrontal areas 
but increasingly also on the prefrontal-basal ganglia loop (e.g., Bogacz and Gurney, 2007; Sieveritz et al., 2019). Moreover, the interaction between the basal ganglia and the frontal regions has been proven in working memory access control: the basal ganglia detects the appropriate context for a motor response to a stimulus stored in memory (McNab and Klingberg, 2008; Baier et al., 2010; Guo et al., 2018).

Working memory is currently viewed as a multi-component system consisting of three subsystems and a central executive one (Baddeley, 2003). These subsystems include the visuospatial sketchpad, which enables storage of visual information, the phonological loop involved in auditory and verbal information storage, and the episodic buffer, which integrates information from other components keeping a continuous sequence. For many years, researchers attempted to understand the neural correlates of information remembering and retrieving. The hippocampus is a neural structure whose participation in both long-term and working memory has been confirmed in many studies (e.g., Olson et al., 2006; Yonelinas, 2013; Libby et al., 2014). It is also well-known that the human memory is prone to errors (Loftus, 1979; Schacter et al., 1998), a fact that motivates the investigation of memory distortion (i.e., false memories) as a byproduct of the memory system, attempting to reveal their nature and mechanism (for meta-analysis on fMRI studies, see: Kurkela and Dennis, 2016). The prevalent paradigm for studying false memories is the DRM (Deese-RoedigerMcDermott) paradigm (Deese, 1959; Roediger and McDermott, 1995). Investigating the neural mechanism of false recognition of verbal or non-verbal stimuli is relevant for both encoding (Kim and Cabeza, 2006) and retrieval phases (Schacter et al., 1998). Regarding the false alarms (i.e., when participants incorrectly claim that a new, similar stimulus has appeared previously), most fMRI studies have been using verbal material (e.g., Kim and Cabeza, 2006; Atkins and Reuter-Lorenz, 2011), while the studies using visual objects are less frequent (Lewandowska et al., 2019; Sikora-Wachowicz et al., 2019, 2021). Neuroimaging studies with pictorial material revealed increased activation in the anterior cingulate cortex (Sikora-Wachowicz et al., 2021) and frontal, parietal, and visual cortices (Slotnick and Schacter, 2004; GaroffEaton et al., 2006; Gutchess and Schacter, 2012) related to false recognitions. In our study, we used two tasks requiring global and local information processing, respectively. The previous neuroimaging research revealed hemispheric differences in activation of brain regions during these two processing strategies, namely global information processing in the right hemisphere and local-in the left hemisphere, and are processed by other networks in these hemispheres (Fink et al., 1996; Martinez et al., 1997; Weissman and Woldorff, 2004).

According to Borbély's two-process model, circadian processes, the endogenous oscillatory pacemaker, and the homeostatic sleep pressure, which increases with time spent awake, regulate overall human performance and behavior during the 24 h cycle (Borbély, 1982; Daan et al., 1984; Borbély et al., 2016). Indeed a large number of studies consistently revealed differences in the cognitive domain according to the time of day (for review, see: Schmidt et al., 2007). Alertness, attention, executive functions, among many others, can be affected by the circadian clock. Also, both short- and long-term memory might be modulated by the time of day (Fabbri et al., 2013; Schmidt et al., 2015).

The present study aims to find neural correlates of encoding and retrieval and the diurnal activity of those correlates in the modified DRM paradigm with abstract, visual objects using a new analysis method-non-linear correlation implemented to the task environment. A typical approach to establish a functional proxy for brain connectivity is to calculate the Pearson linear correlation between the brain's blood oxygenation level dependent (BOLD) time series and a given stimulus of interest. The alternative used here is motivated by the fact that BOLD fluctuations of a relatively large amplitude capture most of the information (Tagliazucchi et al., 2011, 2012; Liu and Duyn, 2013; Petridou et al., 2013). In consequence, bursts of correlated activity across the brain may be efficiently described by a point process consisting of few discrete events (Cifre et al., 2020). The result was subsequently observed with related methods (Liu et al., 2013; Allan et al., 2015; Karahanoğlu and Van De Ville, 2015), and coactivation patterns driven by the point process were studied also in the task paradigm (Jiang et al., 2014; Chen et al., 2015) and in the clinical context (Amico et al., 2014; Li et al., 2014). Following that work, the present correlation approach focuses on the analysis of the brief instances of large-amplitude signals (so-called events); such amplitude thresholding acts as a simple non-linear filter (similarly, to e.g., the effect of using sigmoid activation functions in artificial neural networks), a feature that by design increases the signal-to-noise ratio significantly. This method and its related implementations mentioned above, has been proven effective in analyzing the brain functional connectivity in restingstate conditions (Tagliazucchi et al., 2011, 2012; Cifre et al., 2021), where it is essential to identify the most significant events from the spontaneously fluctuating signals. The present study is the first to implement a similar approach to characterize the responses evoked by a task.

\section{MATERIALS AND METHODS}

\section{Participants}

As many as 5,354 volunteers applied to the first selection stage through the lab's website announcements. All of them were asked to complete a sleep-wake online assessment including diurnal preference-Chronotype Questionnaire (Oginska et al., 2017), night sleep quality-Pittsburgh Sleep Quality Index (PSQI) (Buysse et al., 1989), and daytime sleepiness-Epworth Sleepiness Scale (ESS) (Johns, 1991). Based on the Chronotype Questionnaire, the individuals were divided into morning and evening chronotypes. Then, 451 participants were qualified for the next selection step included genetic testing for the polymorphism of clock gene PER3, which has been established as a hallmark of extreme diurnal preferences (Archer et al., 2003). After selection, fifty-four volunteers participated in the analysis ( 32 females, mean age: $24.17 \pm 3.56$ y.o.) divided into 26 morning types (mean age: $24.31 \pm 3.74$ y.o.) and 28 evening types (mean age: $24.04 \pm 3.24$ y.o.). Exclusion criteria were: age below 19 and above 35, left-handedness (assessed by the 
Edinburgh Handedness Inventory), psychiatric or neurological disorder, drug, alcohol, or nicotine dependence, shift work or travel involving moving between more than two time zones within the past 2 months, and sleep problems (a result above 10 points from ESS caused exclusion). Subjects did not have any contraindications for magnetic resonance imaging studies. The volunteers were remunerated for participation in the experiment. Prior to the completion of study procedures, they were asked to sign a consent form. The study was conducted under the Declaration of Helsinki and approved by the Research Ethics Committee at the Institute of Applied Psychology at the Jagiellonian University, Krakow, Poland.

\section{Task}

The task procedure was based on the DRM paradigm established to investigate the false memories in long-term memory. Given differences between the two types (long- and short-term), the modified version to study short-term memory was developed (Atkins and Reuter-Lorenz, 2011). Two tasks using non-verbal material (abstract, visual objects) requiring global and local information processing were analyzed. The participants had to memorize the set of two stimuli followed by a mask. Subsequently, the stimulus (probe) was displayed, for which a reaction was required, whether the stimulus presented on the screen was present in the preceding set ("yes" with the right hand, "no" with the left). There were three conditions: positive probe (in which the stimulus had been presented in the preceding set), negative probe (the probe had not been presented earlier), and lure (in which the stimulus was very perceptually similar to these in the preceding set but it had not been presented). The third condition seems to produce false memories. Lure probes differed from stimuli in the preceding set in a holistic way (in the "global" task) or individual details (in the "local" task).

There were 60 memory sets presented for $1,800 \mathrm{~ms}$ followed by 25 positive probes, 25 lures, and 10 negative probes presented for $2,000 \mathrm{~ms}$. The memory set and mask were separated by $1,000 \mathrm{~ms}$, whereas the mask and probe by 2,000-16,000 ms (avg. 6,097 ms). The two versions of the tasks were created (for morning and evening sessions); each had six versions of the procedure and was counterbalanced within subjects. The dark gray (RGB 72, 72,72 ) stimuli were presented on a light-gray background (RGB $176,176,176)$. The abstract objects $\left(5^{\circ}\right.$ wide and $4^{\circ}$ high $)$ in memory sets were displayed $3^{\circ}$ from the screen center to the left and right, while masks and the objects in memory probes in the center of the screen. The task was prepared using E-Prime 2.0 (Psychology Software Tools) and performed during fMRI sessions. The previous study (Ceglarek et al., 2021) describes the task in detail. The example task procedure and analysis flow is depicted in Figure 1.

\section{Procedure}

The participants were asked to sleep well (at least $8 \mathrm{~h}$ ) the week before and during the entire experimental period. The duration and quality of sleep in the preceding week were controlled using the MotionWatch8 actigraphs (CamNtech, Cambridge, United Kingdom). The MR acquisition was conducted twice: in the morning and evening session ( 1 and $10 \mathrm{~h}$ after waking up, respectively; cf. Schmidt et al., 2012). The order of sessions was counterbalanced within subjects. Half of the participants started the procedure with the morning session and half with the evening session. The participants spent the night (before or between sessions) in the Małopolska Centre of Biotechnology in Krakow, Poland, in the same building as the fMRI laboratory. Before the proper experiment in the scanner, computer training was conducted to familiarize them with the MR environment and the task. Morning-type participants performed the task between 09:25 $\mathrm{AM}$ and 09:55 AM (SD: $1 \mathrm{~h} 12 \mathrm{~min}$ ) in the morning and between 06:30 PM and 07:02 PM (SD: $1 \mathrm{~h} 26 \mathrm{~min}$ ) in the evening. Evening-type participants performed the task between 11:00 AM and 11:30 AM (SD: $1 \mathrm{~h} 17 \mathrm{~min}$ ) in the morning and between 08:40 PM and 09:10 PM (SD: $1 \mathrm{~h} 07 \mathrm{~min}$ ) in the evening. High variability in the task execution time resulted from four experimental tasks being performed during each session, presented in a semi-random way (for more information about other tasks, see: Lewandowska et al., 2018). Individuals abstained from alcohol $(48 \mathrm{~h})$ and caffeine $(24 \mathrm{~h})$ before the first session and were banned from caffeine and alcohol intake during the experimental days.

\section{Behavioral Data Analysis}

Statistical analyses were performed using Statistica v13.3 (StatSoft, Inc, 2012) software. To observe differences in accuracy (proportion of correct responses) and reaction times (RTs) the generalized linear model (GLM) with accuracy and RTs as dependent variables with sex and chronotype as between-subjects factors, and with time-of-day, response types (correct and false responses to positive, lure and negative probe) and task (global, local) as within-subjects factors was performed. Due to the small number of errors for a negative probe, only the correct recognitions were left for further analysis. The significance level was set at $p<0.05$, multiple-comparison corrected.

\section{fMRI Data Acquisition}

Structural and functional data were collected on a 3T scanner Skyra (Siemens Magnetom, Erlangen, Germany) in Małopolska Centre of Biotechnology in Krakow, Poland, with a 64-channel head coil. For task, 709 functional image volumes with 34 contiguous interleaved axial slices were collected with a T2*weighted echo-planar sequence $(\mathrm{TR}=1,800 \mathrm{~ms}$, $\mathrm{TE}=27 \mathrm{~ms}$, flip angle $=75^{\circ}, \mathrm{FOV}=256 \mathrm{~mm}$, bandwidth: $1,816 \mathrm{~Hz} / \mathrm{Px}$, voxel size: $4 \times 4 \times 4 \mathrm{~mm}$ ). Structural data were acquired for each participant using a T1-weighted MPRAGE sequence for a detailed reconstruction of anatomy with isotropic voxels $(1 \times 1 \times 1.1 \mathrm{~mm})$ in a $256 \mathrm{~mm}$ field of view $(256 \times 256$ matrix, 192 slices, $\mathrm{TR}=2,300 \mathrm{~ms}, \mathrm{TE}=2.98 \mathrm{~ms})$. Stimuli were projected on a screen positioned behind a subject's head; participants viewed the screen in a $45^{\circ}$ mirror fixated on the top of the head coil.

\section{fMRI Preprocessing}

The preprocessing was performed using the Statistical Parametric Mapping software package (SPM12, Welcome Department of 


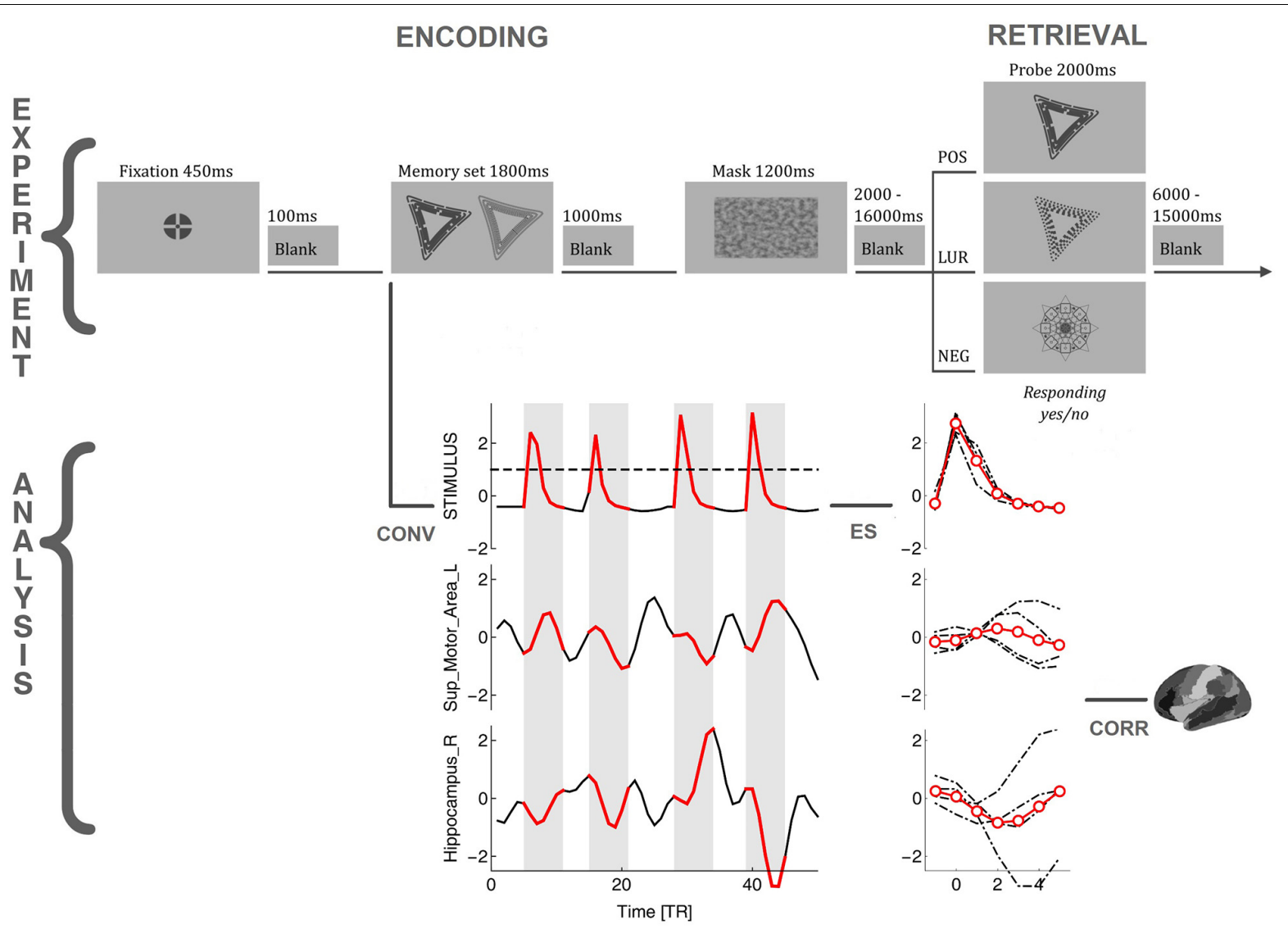

FIGURE 1 | The flowchart of the task requiring global information processing and analysis procedure. POS/LUR/NEG, positive, lure, and negative probes; CONV, convolution of the stimuli with a model hemodynamic response function; ES, identification, extraction, and averaging of BOLD events; CORR, correlating BOLD events with stimuli, Fisher transforming, and performing GLM analysis.

Imaging Neuroscience, UCL, London, United Kingdom $)^{1}$ and DPABI (V4.2; Yan et al., 2016) implemented in MATLAB (Mathworks, Inc., MA, United States). Functional images were slice-time corrected, realigned using rigid body transformation, co-registered, and normalized to the EPI template in Montreal Neurological Institute (MNI) stereotactic space with a voxel resolution $3 \times 3 \times 3 \mathrm{~mm}$. Seven participants were excluded from the research group due to extensive movements exceeding a $4 \mathrm{~mm}$ and/or $4^{\circ}$ on any axis. The data were spatially smoothed using a Gaussian kernel of FWHM $4 \mathrm{~mm}$, detrended and the covariates like motion parameters, mean signal, white matter, and CSF were regressed, following results indicating that global signal regression could be helpful to eliminate potential confounders (Hampson et al., 2010). The signal was then filtered with a 0.01$0.1 \mathrm{~Hz}$ filter, and the time series from 90 regions of interest (ROI) of the AAL atlas were extracted (Tzourio-Mazoyer et al., 2002).

\section{Non-linear Directed Functional Co-activations}

As commented in the introduction, we used a method originally designed to study the correlation between brain regions during

${ }^{1}$ www.fil.ion.ucl.ac.uk/spm/ the brain resting state (i.e., no task). In the classical approach (dynamic functional connectivity), one estimates the resting state correlation by calculating some kind of sliding-window linear Pearson correlation between pairs of BOLD time series. In contrast, the method introduced by Tagliazucchi et al. (2011, 2012) and subsequent authors (Liu and Duyn, 2013; Petridou et al., 2013; Allan et al., 2015; Karahanoğlu and Van De Ville, 2015; Cifre et al., 2020, 2021) relies on detecting for a given source BOLD time series the relatively high amplitude activity ("events") and correlating only these epochs with the other target time series, see Figure 1. The amplitude threshold, or Heaviside step function, is in fact a very simple non-linear filter (akin to the sigmoid functions used, e.g., as a non-linear activation in artificial neural networks) used for signal denoising. The approach is naturally connected to the hemodynamic response function (HRF; Wu et al., 2013) and has allowed to demonstrate the correspondence between rest and task BOLD activations (Petridou et al., 2013). Additionally, such a procedure provides the correlations with a straightforward directionality and timestamps (Cifre et al., 2021). Thanks to these features, we were able to apply the method to the task setting with the series of task stimuli serving as a synthetic source time series, as described below. 


\section{Definition of Source and Target Events}

First, as depicted in Figure 1, the times of stimuli appearance were determined for the Memory sets (encoding phase) and Probes (retrieval phase). Next, to generate a time series encoding the stimuli appearance, we created a binary variable with the sampling rate of $1 \mathrm{kHz}$ (higher than the accuracy of stimulus timing measurements from E-Prime 2.0). Then this time series was convolved with the model HRF obtained from SPM12. After that, it was resampled to 1/TR to align it with the actual BOLD signal, and the resulting time series (termed here stimuli time series) was normalized by its standard deviation (i.e., z-scored). In the present approach, the correlation is computed between the "source" and the "target" events. The "source" events were extracted from the stimuli time series, as segments of 5 TRs after the signal crossed the threshold of 1, including the crossing itself, which is enough to represent the HRF's whole positive peak. The amplitude thresholding is essential in the restingstate analysis, where the events have to be first identified in the signal to subsequently perform the pairwise correlations. In the present task design, the non-linear filter action of the peak selection mostly helps to synchronize TRs with the timing of the stimuli. For the computation of time delays between stimuli and the BOLD signal, the peak selection introduces a similar nonlinearity. Finally, the "target" events are extracted from the BOLD time series (of each of the 90 AAL ROIs; the atlas regions with MNI coordinates are presented in the Supplementary Table 1) at precisely the same times as the "source" events. To investigate the influence of motion artifacts on the events' selection, we have checked that there are only 5 motion events in GLO task and 3 events in LOC task larger than $4 \mathrm{~mm}$ across all participants (with $4 \mathrm{~mm}$ being the voxel size). Even if we take $1 \mathrm{~mm}$ events ( $1 / 4$ voxel size), only $0.64 \%$ in GLO (and $0.60 \%$ in LOC) of them overlap with the stimulus events (when the events are $0-$ 9 TRs around the stimulus), so we believe that influence of so few events is negligible.

\section{Correlations}

The linear Pearson correlation between source and target events was computed and averaged for each experimental condition (subject, session time, phase, probe type, response, and ROI), and the averages were Fisher transformed. The values of these correlations indicate whether a particular ROI systematically co-activated (positive correlations) or deactivated (negative correlations) with a given stimulus type in a given condition.

\section{Delays}

Conventionally, the delay between a pair of BOLD time series is defined by the lag at which lagged cross-covariance between them has an extremum. Such a delay has the resolution of multiple integer of TRs. For a finer resolution, a parabolic interpolation of the extremum can be used (Mitra et al., 2014). In the approach by Cifre et al. (2021), which we used, the delay is defined as the time between the peak of each individual BOLD-triggered event (substituted in the task paradigm by the synthetic stimulus HRF convolutions) and the closest BOLD peak irrespective of its size. Given the objective of the present study, we looked for the peaks in the range $[0,9]$ TRs (viz. only after the stimulus). We used the parabolic peaks fitted to 3 TRs for finer resolution. Note that the parabolic estimation at the edges of the interval may lead to both positive and negative outliers outside the interval. Consequently, we discard values outside $[-1,10]$.

\section{fMRI Data Analysis}

Statistical analyses were performed using the $\mathrm{R}$ stats package ( $\mathrm{R}$ Core Team, 2021) and the estimated marginal mean package emmeans (Lenth, 2021); the data and R scripts are provided in the Supplementary Material. The general linear model (GLM) assumed response type ("yes," "no") as the predicted variable, the phase (retrieval, encoding), probe (positive, lure; the negative probe was not used due to the small number of errors, resulting in no predictive value), condition (morning, evening) and $R O I$ (90 AAL regions) as the nominal predictors, and the average correlations as the numerical predictor. Note that we did not use sex and chronotype variables, present in the behavioral analysis, due to the prohibitive size of the full model; the results of a smaller model (without ROIs) can be found in Supplementary Material. Consequently, we used logistic regression with up to four-way interactions correlation $\times R O I \times$ (all pairs in the set: phase, probe, condition), but a priori excluding the terms: phase, ROI and their interactions with probe and condition, since responses are independent of their levels. Such a model was further reduced by a single term deletion based on the Akaike information criterion; the reduction was finished when the variable or interaction, whose deletion minimized AIC, was at the same time significant with $p<0.05$. The significance level was set at $p<0.05$, multiple-comparison corrected (Sidak adjustment). The presented results are estimates transformed back from logit to the original variables.

\section{RESULTS}

\section{Behavioral Data}

The GLM with accuracy and RTs as dependent variables with sex and chronotype as between-subjects factors, and with timeof-day, response types (correct and false responses to positive, lure and negative probe) and task (global, local) as withinsubjects factors revealed significant influence of response type $\left[F(1,8)=439 ; p<0.001 ; \eta_{p}^{2}=0.64\right]$ and interactions: probe $\times$ task $\left[F(1,8)=21,47 ; p<0.001 ; \eta_{p}^{2}=0.08\right]$

TABLE 1 | The proportion of responses and reaction times for response types in both tasks.

\begin{tabular}{lcccc} 
Probe type & Response type & Proportion of responses & Reaction times (ms) \\
\cline { 3 - 3 } Positive & Hits & Mean \pm SD & & Mean \pm SD \\
Lure & Misses & $0.82 \pm 0.13$ & & $1,277 \pm 226$ \\
& Correct rejections & $0.74 \pm 0.19$ & & $1,295 \pm 201$ \\
Negative & False alarms & $0.25 \pm 0.17$ & & $1,392 \pm 419$ \\
& Correct rejections & $0.98 \pm 0.08$ & & $992 \pm 161$ \\
& False alarms & $0.02 \pm 0.04$ & & $1,350 \pm 264$
\end{tabular}




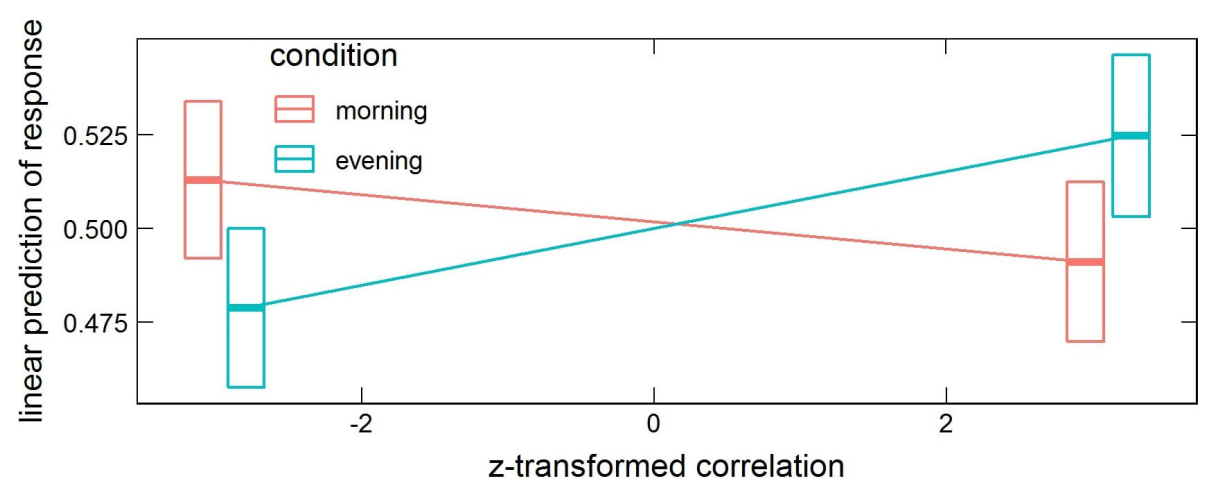

FIGURE 2 | Estimated means' interaction condition $\times$ correlation in the global processing task, averaged over phase, probe and ROI levels. Horizontal bars are $95 \%$ Cls.

and sex $\times$ chronotype $\times$ probe $[F(1,8)=2.70 ; p=0.006 ;$ $\left.\eta_{p}^{2}=0.01\right)$. The post hoc HSD Tukey test for accuracy indicated the differences between all response types; for RTs-also between all response types except correct recognition of positive probe vs. correct rejection of lure probe and false responses for positive and lure probes. The descriptive statistics on the proportion of responses and reaction times are presented in Table $\mathbf{1}$.

\section{fMRI Data Analysis}

The GLMs predicting response type ("yes," "no") based on condition, phase, probe type, correlation, and ROI were fitted separately for the global and local processing tasks. The complete type III ANOVA tables for each model are in the Supplementary Material. Below we report in detail only the highest order significant interactions. The sex and chronotype were omitted from the model with ROI interactions, however these variables jointly with the condition might have an additional modulating effect (a smaller model provided in the Supplementary Material yields interactions condition $\times$ correlation $\times$ sex $\times$ chronotype $\times$ phase and condition $\times$ probe $\times$ correlation $\times$ sex $\times$ phase in GLO and condition $\times$ probe $\times$ correlation $\times$ chronotype $\times$ phase in LOC task).

\section{Task Requiring Global Information Processing}

The model revealed significant interactions: condition $\times$ correlation $\left(\chi^{2}=5.26, d f=1, p=0.022\right)$ and correlation $\times$ phase $\times$ probe $\times R O I\left(\chi^{2}=332.36, d f=89\right.$, $\left.p<2.2 \times 10^{-16}\right)$. In the first case, the contrast between trends of response as a function of correlation in the evening and morning conditions (averaged over all phases, probes, and ROIs) was estimated to be $0.014(p=0.022)$, with the small effect of correlation increasing the chance of saying "no" in the evening and of saying "yes" in the morning (morning 95\% CI $[-0.014$, 0.0032] and evening 95\% CI [0.00013, 0.017]), see Figure 2.

The interaction correlation $\times$ phase $\times$ probe $\times R O I$ disclosed several significant ROIs in encoding and retrieval phases (effects presented in Table 2 and Figure 3). The increasing correlation of hippocampal areas with retrieval stimulus predicted more "no" responses in the positive probe and fewer in the lure. A reverse effect was observed for the left supplementary motor area (retrieval), olfactory, and medial orbitofrontal cortex (encoding). These brain regions are displayed in Figure 4A.

\section{Task Requiring Local Information Processing}

In the local processing task, the highest order significant interaction containing condition was: condition $\times$ probe $\times$ correlation $\times$ phase $\left(\chi^{2}=9.90, d f=1\right.$, $p=0.0017)$. Similarly to the global processing task, the interaction correlation $\times$ phase $\times$ probe $\times R O I\left(\chi^{2}=994.45\right.$, $d f=89, p<2.2 \times 10^{-16}$ ) was also found significant.

In the interaction condition $\times$ probe $\times$ correlation $\times$ phase the contrast morning-evening was revealed significant for positive probe in the encoding phase, 95\% CI $[0.017,0.070](p=0.0051)$, and for lure probe in the retrieval phase, 95\% CI [0.026, 0.078 ] $(p=0.00043)$, as well as the contrast lure-positive probe in the evening for both encoding, 95\% CI [-0.085, $0.0024](p=0.000037)$, and retrieval, 95\% CI [0.0020, 0.087] $(p=0.000066)$. These results are depicted in Figure 5.

In the interaction correlation $\times$ phase $\times$ probe $\times R O I$, 18 significant ROIs in encoding and 17 in retrieval phases were disclosed (Table 3). The six most significant brain regions for the encoding (left and right anterior cingulate

TABLE 2 | Estimated means' interaction correlation $\times$ phase $\times$ probe $\times$ ROI in the global processing task, averaged over condition levels.

\begin{tabular}{cccccc}
\hline & ROI & LUR-POS & SE & z ratio & p-value \\
\hline Encoding & & & & & \\
\hline \multirow{2}{*}{ Retrieval } & Olfactory_L & 0.33 & 0.078 & 4.26 & 0.0037 \\
& Frontal_Med_Orb_L & 0.29 & 0.078 & 3.70 & 0.038 \\
& & 0.31 & 0.071 & 4.36 & 0.0023 \\
& Supp_Motor_Area_L & 0.33 & 0.078 & 4.26 & 0.0037 \\
& Hippocampus_R & -0.31 & 0.084 & -3.71 & 0.037 \\
& ParaHippocampal_L & -0.31 & 0.080 & -3.94 & 0.015 \\
& ParaHippocampal_R & -0.33 & 0.082 & -4.02 & 0.010 \\
\hline
\end{tabular}

We present the ROls, where the contrast lure-positive yielded $p<0.05$; $p$-values are corrected (Sidak adjustment) see Figure 3. Results of all the ROIs are plotted in Supplementary Figure 1. 


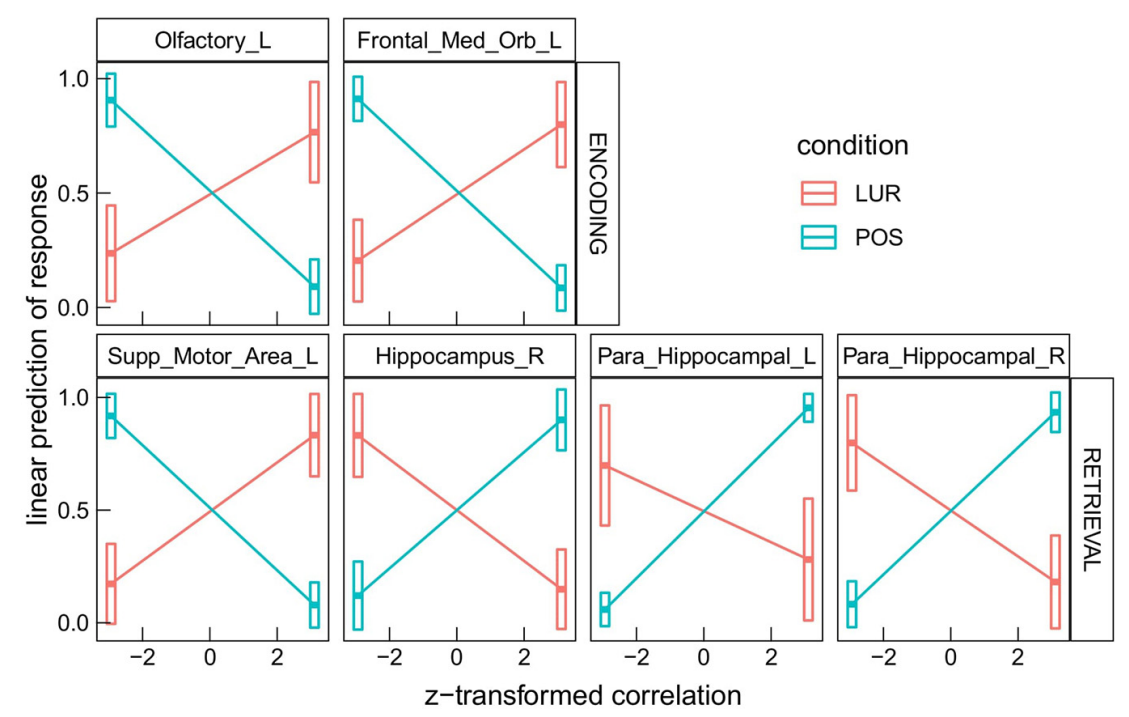

FIGURE 3| Results of the correlation $\times$ phase $\times$ probe $\times$ ROI interaction in the global processing task, averaged over condition levels. Horizontal bars are $95 \%$ Cls.

cortex, left precentral gyrus, left superior frontal gyrus, left superior frontal gyrus medial part, right medial frontal gyrus orbital part) and the retrieval phase (left and right supplementary motor area, right hippocampus, superior temporal pole and left inferior frontal gyrus) are displayed in Figure 4B. The above results are supplemented with Figure 6, showing the distributions of individual BOLD peak delays from the synthetic stimulus HRF convolutions as measured in the left supplementary motor area, right inferior frontal gyrus and right hippocampus. The delay distributions for all the other ROIs are presented in Supplementary Figures 3, 4. The distributions were collected from all the subjects and conditions not explicitly mentioned in the figures (i.e., time-of-day, response type or probe type). The noteworthy features are the number of distribution modes and their location.

\section{DISCUSSION}

The current study investigated false memory formation in short-term memory. Employing a novel approach, it revealed features of the neural mechanism behind memory distortions. Although the method has been applied before to resting-state data, according to our knowledge, the present results are the first attempt to employ this technique to task fMRI data. We successfully demonstrate the use of the non-linear correlation method on short-term memory tasks requiring global and local visual processing as well as search for diurnal differences in correlations.

These results indicate some time-of-day effects on the neural mechanism of false memories formation. Previous studies revealed time-of-day differences in neural activity during cognitive tasks (for a review, see: Gaggioni et al., 2014). Marek et al. (2010) confirmed diurnal variations in neural activity of orienting the attentional system during a Strooplike task. Schmidt et al. (2015), using the n-back paradigm with different cognitive load, demonstrated decreased activity in the ventrolateral prefrontal cortex and premotor areas from the morning to the evening hours for the higher cognitive load. The interaction of time-of-day with BOLD correlations that we observe are in general weaker than other ones (e.g., correlation $\times$ probe). In the global processing task, the overall correlations with stimuli predict a higher proportion of "no" responses in the evening and lower in the morning. However, other interactions (with phase and probe), which appeared significant only in the analysis of the local processing task, might confound this effect. Nevertheless, our result with diurnal variation of responses is consistent with the recent work of Tandoc et al. (2021), which revealed an increased generalization process that leads to increased false memory formation in the morning-an effect explained by lower inhibition at morning hours. Another study on resting-state data suggested the less efficient brain networks organization in the first hours after waking, which could be an effect of sleep inertia (Farahani et al., 2021). The stronger effect for local processing, visible in Figure 5, reads that in the evening the increased correlation of the whole brain's activity with encoding stimulus predicts a lower proportion of "no" responses to a positive probe (more correct responses), and similarly, the increased correlation with retrieval stimulus predicts a lower proportion of "no" responses to a lure probe (more incorrect responses).

Regions that showed significant differences in correlations in both tasks overlapped in the retrieval phase (see: Tables 2, 3), which strengthens the result and demonstrates the effectiveness of the new method of analysis. Regarding the task requiring global information processing, we observed differences in correlations in the orbitofrontal region for the memorizing phase of the task and hippocampal and parahippocampal areas for the retrieval phase. The orbitofrontal cortex is involved in the process of 
A
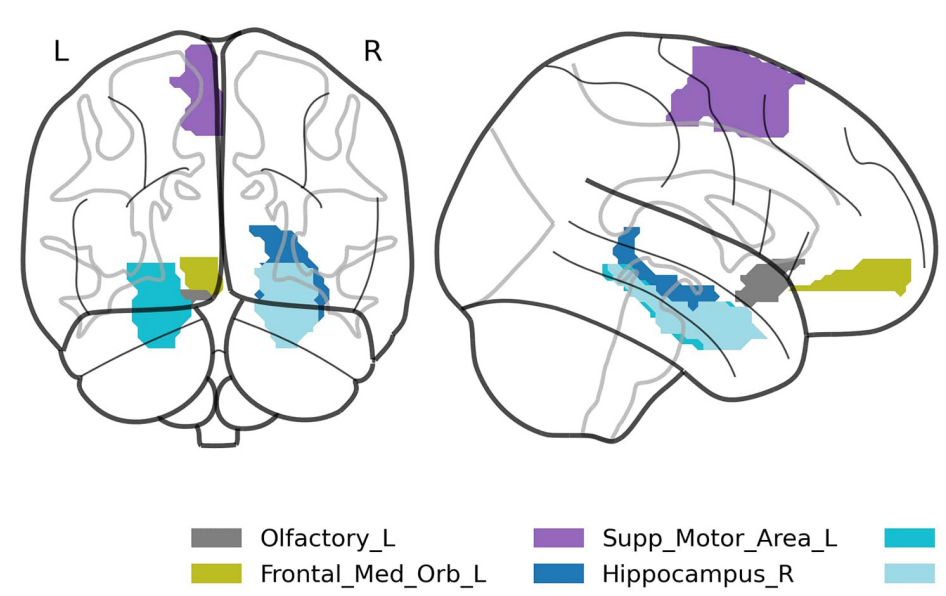

B
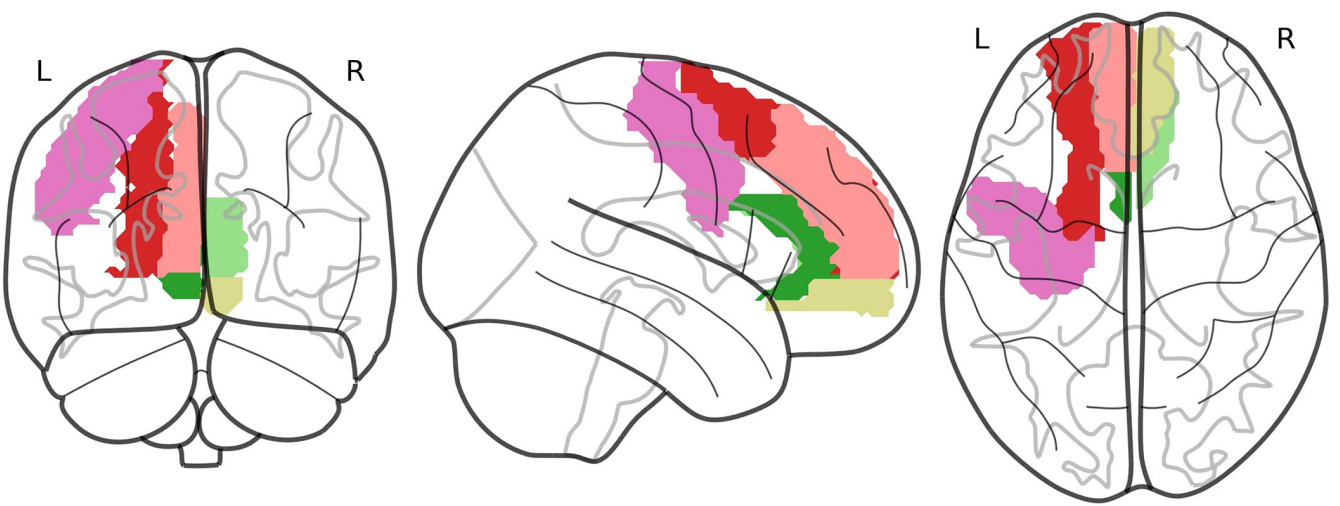

Cingulum_Ant_R

Cingulum_Ant_L

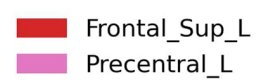

Frontal_Sup_Medial_L

Frontal_Med_Orb_R

C
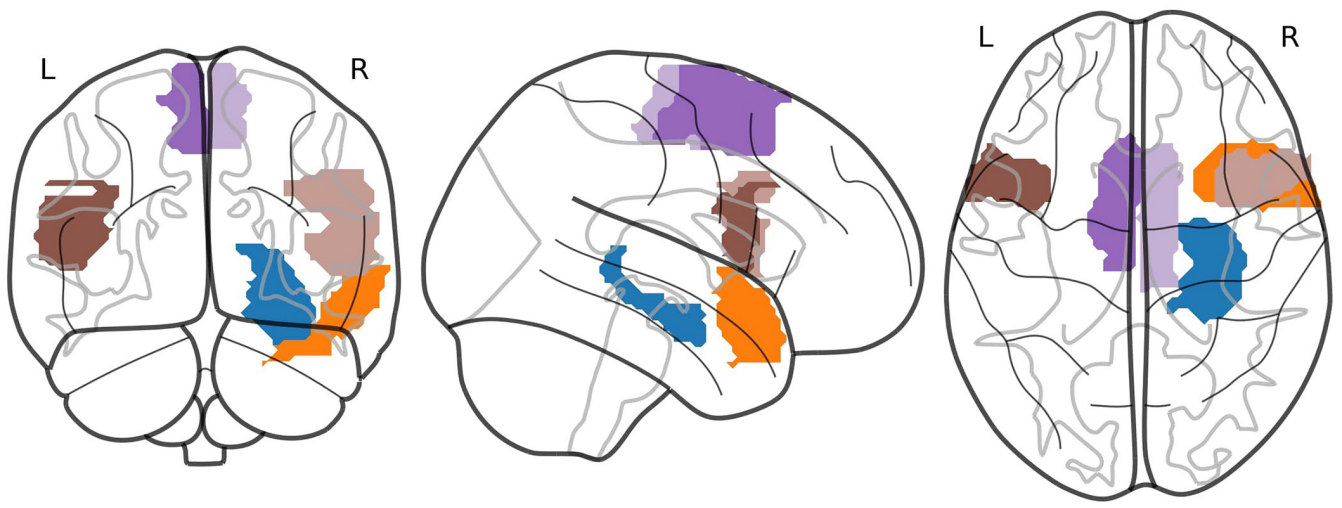

Temporal_Pole_Sup_R
Hippocampus_R

Frontal_Inf_Oper_R
Frontal_Inf_Oper_L

Supp_Motor_Area_R

Supp_Motor_Area_L

FIGURE 4 | Visualization of brain regions whose correlation with the stimuli predicts difference in response between /ure and positive probes in: (A) global information processing task, (B) encoding in local information processing task, (C) retrieval in local information processing task.

decision making (Steiner and Redish, 2012) and encoding the new visual stimuli (Frey and Petrides, 2000; O'Doherty, 2007). The middle temporal cortex, which includes the hippocampus and parahippocampal gyrus, plays a crucial role in remembering and retrieving events, facts, and details. Moreover, using auditory verbal material, Cabeza et al. (2001) suggested that 


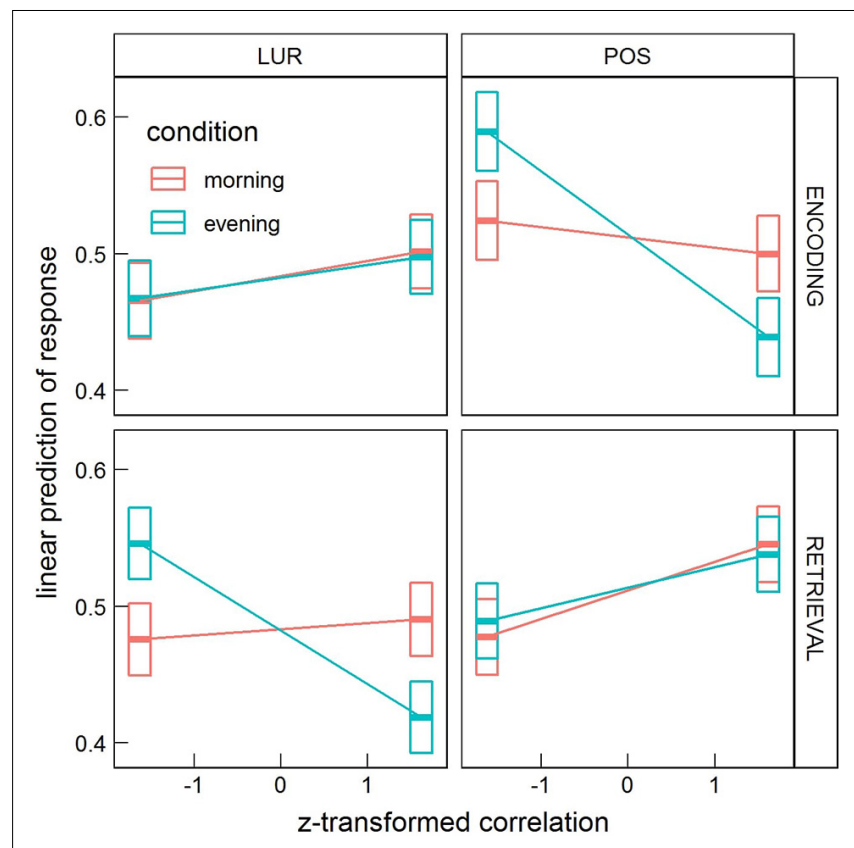

FIGURE 5 | Estimated means' interaction condition $\times$ probe $\times$ correlation $\times$ phase in the local processing task, averaged over condition levels. Horizontal bars are $95 \% \mathrm{Cls.}$

the parahippocampal gyrus can distinguish between false and true recognition. Garoff-Eaton et al. (2006) confirmed that capacity of the right parahippocampal gyrus with a procedure that uses abstract visual stimuli. The current study supports these results, showing that correlations of the right hippocampus and parahippocampal gyri with the positive and lure probe differentially predict subjects' responses. Higher correlation in all these areas consistently predicted more "no" responses to positive probe and fewer for lure (both increasing the proportion of incorrect responses, see Figure 3). The opposite trend was seen in supplementary motor area, which might be due the different nature of this region, not involved specifically in memory processing, but in motor planning (Welniarz et al., 2019).

There are more differences in correlations in the task requiring local (detailed) information processing, especially in frontal, cingulate, and temporal cortices. For the encoding phase, one sees the activations of the anterior, middle, and posterior cingulate cortex, which are part of the limbic system responsible for regulating emotion, learning, and memory (Rolls, 2019). Extensive psychological studies on the functional organization of the brain revealed the hemispheric functional separation in a way that the left hemisphere is engaged in language processing and the right is responsible for visuospatial functions (e.g., Gazzaniga et al., 1965; Milner, 1971; Corballis et al., 1999, 2002; Zuanazzi and Cattaneo, 2017). Our task employing abstract objects located in space seems to engage the right hemisphere more, which can be seen in differences in correlations in right temporal cortices.

We have limited the current study by the choice of the AAL atlas, which did not allow the examination of fine-grained structures such as the hippocampus. The choice was made due
TABLE 3 | Results of the correlation $\times$ phase $\times$ probe $\times$ ROI interaction in the local processing task, averaged over condition levels.

\begin{tabular}{lcccc}
\hline ROI & LUR-POS & SE & z ratio & p-value \\
\hline Encoding & & & & \\
\hline Precentral_L & -0.45 & 0.095 & -4.7 & 0.00045 \\
Precentral_R & -0.34 & 0.092 & -3.7 & 0.032 \\
Frontal_Sup_L & 0.45 & 0.085 & 5.3 & $1.70 \mathrm{E}-05$ \\
Frontal_Sup_R & 0.39 & 0.094 & 4.1 & 0.0072 \\
Frontal_Sup_Medial_L & 0.43 & 0.082 & 5.3 & $2.50 \mathrm{E}-05$ \\
Frontal_Sup_Medial_R & 0.37 & 0.087 & 4.3 & 0.0036 \\
Frontal_Med_Orb_L & 0.34 & 0.083 & 4.1 & 0.0077 \\
Frontal_Med_Orb_R & 0.39 & 0.085 & 4.6 & 0.00075 \\
Cingulum_Ant_L & 0.41 & 0.084 & 4.9 & 0.00014 \\
Cingulum_Ant_R & 0.52 & 0.095 & 5.4 & $9.10 \mathrm{E}-06$ \\
Cingulum_Post_L & 0.39 & 0.08 & 4.8 & 0.00026 \\
Cingulum_Post_R & 0.34 & 0.082 & 4.2 & 0.0046 \\
Para_Hippocampal_R & 0.35 & 0.093 & 3.8 & 0.030 \\
Parietal_Inf_L & -0.35 & 0.084 & -4.2 & 0.0058 \\
Parietal_Inf_R & -0.36 & 0.089 & -4.1 & 0.0082 \\
Temporal_Pole_Sup_R & 0.38 & 0.093 & 4.1 & 0.0074 \\
Temporal_Mid_R & 0.34 & 0.091 & 3.7 & 0.041 \\
Temporal_Pole_Mid_R & 0.38 & 0.091 & 4.1 & 0.0067 \\
Retrieval & & & & \\
Precentral_L & 0.42 & 0.087 & 4.8 & 0.00027 \\
Frontal_Mid_L & 0.41 & 0.093 & 4.4 & 0.0017 \\
Frontal_Mid_Orb_L & 0.32 & 0.085 & 3.8 & 0.027 \\
Frontal_Inf_Oper_L & 0.5 & 0.097 & 5.2 & $3.90 \mathrm{E}-05$ \\
Frontal_Inf_Oper_R & 0.49 & 0.11 & 4.4 & 0.0016 \\
Supp_Motor_Area_L & 0.57 & 0.09 & 6.3 & $6.00 \mathrm{E}-08$ \\
Supp_Motor_Area_R & 0.54 & 0.1 & 5.3 & $2.20 \mathrm{E}-05$ \\
Hippocampus_L & -0.38 & 0.093 & -4.1 & 0.0065 \\
Hippocampus_R & -0.51 & 0.097 & -5.3 & $2.60 \mathrm{E}-05$ \\
Para_Hippocampal_R & -0.44 & 0.087 & -5 & $9.00 \mathrm{E}-05$ \\
Amygdala_R & -0.39 & 0.087 & -4.5 & 0.0013 \\
Cuneus_L & -0.37 & 0.087 & -4.3 & 0.0036 \\
Parietal_Sup_L & 0.42 & 0.095 & 4.4 & 0.0018 \\
Temporal_Sup_L & -0.39 & 0.095 & -4.1 & 0.0089 \\
Temporal_Pole_Sup_L & -0.36 & 0.092 & -4 & 0.012 \\
Temporal_Pole_Sup_R & -0.53 & 0.098 & -5.4 & $9.20 \mathrm{E}-06$ \\
Temporal_Pole_Mid_R & -0.34 & 0.09 & -3.7 & 0.032 \\
\hline PresenttheRORIs,wethe & & & & \\
\hline & & & & \\
\hline
\end{tabular}

We present the ROls, where the contrast lure-positive yielded $p<0.05$; $p$-values are corrected (Sidak adjustment). Six regions having the largest effect correlated with encoding and retrieval stimulus are in bold, and are presented in Figures 4B,C.

to partly exploratory character of the present analysis and for the sake of presenting a proof of concept. Nonetheless, there are no technical limitations to apply the present approach to a much finer atlas or even to specific voxels (especially with a hypothesis driven analytic design, where not all brain areas are of interest).

It is worth mentioning that the type of correlation analysis used here is appealing, given the recent results indicating that BOLD infraslow signal fluctuations throughout the brain are coherent with arousal fluctuations (Raut et al., 2021). These results on humans fMRI show that ongoing arousal fluctuations are correlated with global waves of activity slowly propagating in parallel through the neocortex, thalamus, striatum, and 

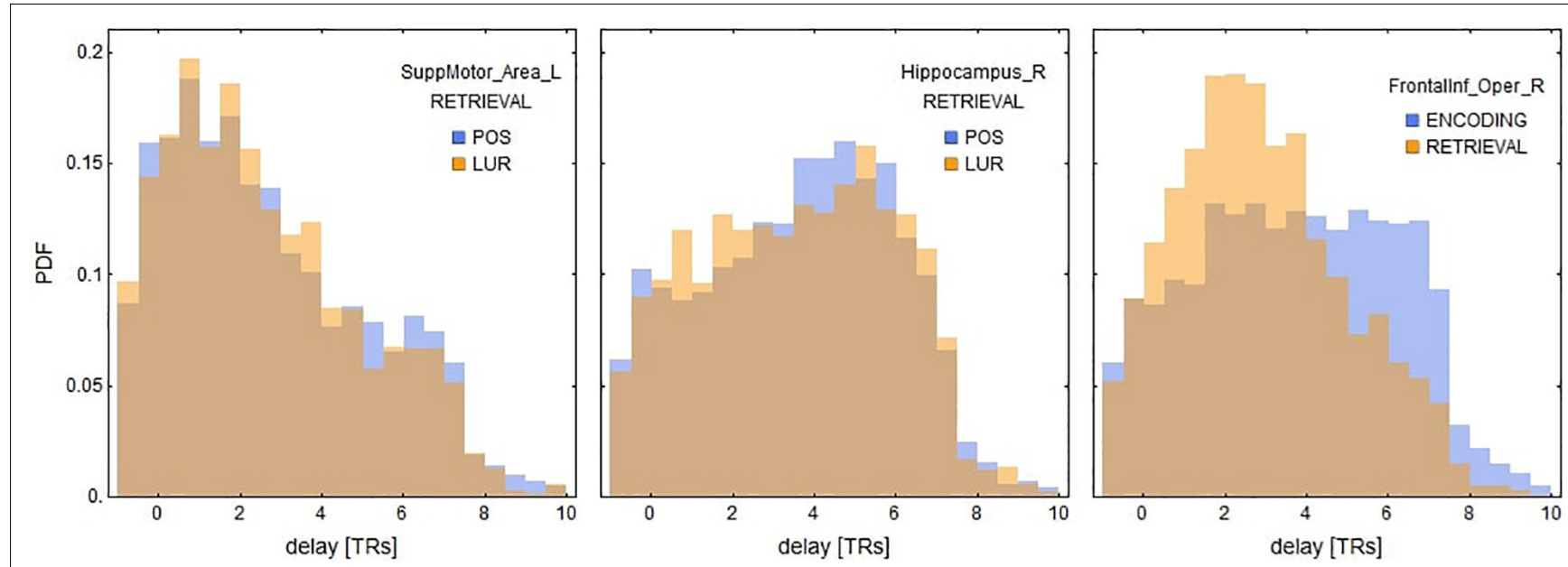

FIGURE 6 | Distributions of delays between the stimuli and the nearest BOLD peaks for three ROls significant for retrieval in local information processing (see Table 3). These results suggest that the positive correlations of supplementary motor areas and inferior frontal gyri might be explained by the shorter delays, whereas negative correlations of hippocampal areas by relatively longer ones. See also Supplementary Figures 3, 4 for the delay distributions of all AAL ROls.

cerebellum. We could speculate that the observed differences between time-of-day may be causally related with these high amplitude waves, corresponding with different degrees of arousal and thus of cognitive performance, a point that deserves further consideration.

This study was the first attempt at implementing the new non-linear co-activations method in the task environment. The limitation of the current application of the method is the possible delay of the BOLD response to the stimuli with respect to the timing of the stimuli themselves. If the dominant peak of the response is 1-5 TRs delayed, then one would observe a negative non-linear correlation with an ROI that, in fact, responds positively to a stimulus, unless the anticipatory vasodilation comes into play (Sirotin and Das, 2009) and synchronizes the BOLD activations. The precise interpretation of an ROI's role following such statements as "higher correlation with an ROI predicts fewer correct responses" is thus contingent on that delay. Incorporating delays into the non-linear correlation analysis is actually possible, as shown in Cifre et al. (2021). In this study, we provide only a proof of concept of that additional feature, a topic that deserves further investigation.

\section{CONCLUSION}

In conclusion, the models generally explained less variance in the global visual-feature processing task data than the local one. The dependence of false memories formation on time-of-day was generally present but weaker than other effects, currently not allowing to pinpoint any particular ROI affected by it. However, diurnal variation of responses could be explained by lower cortical inhibition immediately after sleep, according to the synaptic homeostasis hypothesis. On the other hand, there was enough evidence to find significant differences in processing the positive and the misleading stimuli in specific brain areas. Most notably, we found that peaks in the BOLD signal in the supplementary motor areas immediately after presenting stimuli in the retrieval phase consistently predicted correctness of the following response ("no" for lure and "yes" for positive), and the reverse was true for the hippocampal regions (peaking BOLD at the time of retrieval consistently predicted incorrect responses).

The study demonstrates that non-linear fMRI correlations can be applied effectively to the task paradigm. They were found informative as predictors in generalized linear models, where the interaction terms with atlas-based ROIs indicated specific loci associated with producing responses to the tasks. The method allows finding brain areas related to processing the stimuli and opens new possibilities for analyzing other cognitive tasks. Our results on the influence of the time-of-day on the correlations between the stimulus and neuronal activity and, consequently, on the formation of memory distortions show that the diurnal factor is crucial in various cognitive functions and cannot be ignored when designing research procedures. In addition to scientific research, an important general conclusion from this study is that the human brain functions differently depending on the time of day, which is confirmed in an increasing number of psychological domains.

\section{DATA AVAILABILITY STATEMENT}

The datasets presented in this study can be found in online repositories. The names of the repository/repositories and accession number(s) can be found in the article/Supplementary Material.

\section{ETHICS STATEMENT}

The studies involving human participants were reviewed and approved by the Research Ethics Committee at the Institute of Applied Psychology at the Jagiellonian University, Krakow, 
Poland. The participants provided their written informed consent to participate in this study.

\section{AUTHOR CONTRIBUTIONS}

MF, BS-W, KL, and TM conceptualized the experiment and fMRI tasks procedure. AC, JO, IC, and DC conceptualized the method of the analysis. MF, BS-W, and KL collected the behavioral and imaging data. AC performed the preprocessing steps. AC and JO analyzed the fMRI data and prepared figures. DC provided feedback on results' interpretation. AC, JO, IC, and DC wrote the first draft of the manuscript. All authors reviewed the manuscript.

\section{FUNDING}

This study was funded by the Polish National Science Centre through grants: Harmonia 2013/08/M/HS6/00042 (MF), Sonata 2015/17/D/ST2/03492 (JO), and supported by the Foundation for Polish Science (FNP) project "Bio-inspired Artificial Neural Networks" (POIR.04.04.00-00-14DE/18-00). The open access license of the publication was funded by the Priority Research Area Society of the Future under the program "Excellence Initiative - Research University" at the Jagiellonian University in Kraków.

\section{REFERENCES}

Allan, T. W., Francis, S. T., Caballero-Gaudes, C., Morris, P. G., Liddle, E. B., Liddle, P. F., et al. (2015). Functional connectivity in MRI is driven by spontaneous BOLD events. PLoS One 10:e0124577. doi: 10.1371/journal.pone. 0124577

Amico, E., Gomez, F., Perri, C. D., Vanhaudenhuyse, A., Lesenfants, D., Boveroux, P., et al. (2014). Posterior cingulate cortex-related co-activation patterns: a resting state fmri study in propofol-induced loss of consciousness. PLoS One 9:e100012. doi: 10.1371/journal.pone.0100012

Archer, S. N., Robilliard, D. L., Skene, D. J., Smits, M., Williams, A., Arendt, J., et al. (2003). A length polymorphism in the circadian clock gene per3 is linked to delayed sleep phase syndrome and extreme diurnal preference. Sleep 26, 413-415. doi: 10.1093/sleep/26.4.413

Atkins, A. S., and Reuter-Lorenz, P. A. (2011). Neural mechanisms of semantic interference and false recognition in short-term memory. NeuroImage 56, 1726-1734. doi: 10.1016/j.neuroimage.2011.02.048

Baddeley, A. (2003). Working memory: looking back and looking forward. Nat. Rev. Neurosci. 4, 829-839. doi: 10.1038/nrn1201

Baier, B., Karnath, H.-O., Dieterich, M., Birklein, F., Heinze, C., and Muller, N. G. (2010). Keeping memory clear and stable-the contribution of human basal ganglia and prefrontal cortex to working memory. J. Neurosci. 30, 9788-9792. doi: 10.1523/jneurosci.1513-10.2010

Bogacz, R., and Gurney, K. (2007). The basal ganglia and cortex implement optimal decision making between alternative actions. Neural Comput. 19, 442-477. doi: 10.1162/neco.2007.19.2.442

Borbély, A. A. (1982). A two process model of sleep regulation. Hum. Neurobiol. 1, 195-204.

Borbély, A. A., Daan, S., Wirz-Justice, A., and Deboer, T. (2016). The two-process model of sleep regulation: a reappraisal. J. Sleep Res. 25, 131-143. doi: 10.1111/ jsr.12371

Buysse, D. J., Reynolds, C. F., Monk, T. H., Berman, S. R., and Kupfer, D. J. (1989). The Pittsburgh sleep quality index: a new instrument for psychiatric practice and research. Psychiatry Res. 28, 193-213. doi: 10.1016/0165-1781(89)90047-4

\section{ACKNOWLEDGMENTS}

This work was conducted under the auspice of the Jagiellonian University-UNSAM Cooperation Agreement. AC, BB and JO thanks DC for facilitating their visit and the hospitality of the Universidad de San Martín, UNSAM, Argentina. We would like to thank Patricia Reuter-Lorenz for her constructive suggestions during the planning and development of the project and her valuable support. We also thank Anna Beres and Monika Ostrogorska for their assistance in data collection, Piotr Faba for his technical support on the project and help in data acquisition, and Aleksandra Zyrkowska for help in the process of participants selection. This manuscript was deposited as a preprint at bioRxiv (https://doi.org/10.1101/2021.09.12.459960).

\section{SUPPLEMENTARY MATERIAL}

The Supplementary Material for this article can be found online at: https://www.frontiersin.org/articles/10.3389/fnins. 2021.778242/full\#supplementary-material

The MATLAB code accompanying the manuscript (Cifre et al., 2021) for computing non-linear functional co-activations is available at https://github.com/remolek/NFC. The data, R scripts, fitted models, and results used in statistical analysis in this manuscript are available at https://osf.io/r46wf, as well as supplementary figures.

Cabeza, R., Rao, S. M., Wagner, A. D., Mayer, A. R., and Schacter, D. L. (2001). Can medial temporal lobe regions distinguish true from false? An event-related functional MRI study of veridical and illusory recognition memory. Proc. Natl. Acad. Sci. U. S. A. 98, 4805-4810. doi: 10.1073/pnas.081082698

Ceglarek, A., Hubalewska-Mazgaj, M., Lewandowska, K., Sikora-Wachowicz, B., Marek, T., and Fafrowicz, M. (2021). Time-of-day effects on objective and subjective short-term memory task performance. Chronobiol. Int. 38, 13301343. doi: 10.1080/07420528.2021.1929279

Chen, J. E., Chang, C., Greicius, M. D., and Glover, G. H. (2015). Introducing co-activation pattern metrics to quantify spontaneous brain network dynamics. NeuroImage 111, 476-488. doi: 10.1016/j.neuroimage.2015. 01.057

Cifre, I., Miller Flores, M. T., Ochab, J. K., and Chialvo, D. R. (2021). Revisiting non-linear functional brain co-activations: directed, dynamic and delayed. Front. Neurosci. 15:700171. doi: 10.3389/fnins.2021.700171

Cifre, I., Zarepour, M., Horovitz, S. G., Cannas, S. A., and Chialvo, D. R. (2020). Further results on why a point process is effective for estimating correlation between brain regions. Papers Phys. 12:120003. doi: 10.4279/pip.120003

Corballis, P. M., Funnell, M. G., and Gazzaniga, M. S. (1999). A dissociation between spatial and identity matching in callosotomy patients. NeuroReport 10 , 2183-2187. doi: 10.1097/00001756-199907130-00033

Corballis, P. M., Funnell, M. G., and Gazzaniga, M. S. (2002). Hemispheric asymmetries for simple visual judgments in the split brain. Neuropsychologia 40, 401-410. doi: 10.1016/s0028-3932(01)00100-2

Daan, S., Beersma, D. G., and Borbély, A. A. (1984). Timing of human sleep: recovery process gated by a circadian pacemaker. Am. J. Physiol. Regul. Integr. Comp. Physiol. 246, 161-183. doi: 10.1152/ajpregu.1984.246.2.r161

Deese, J. (1959). On the prediction of occurrence of particular verbal intrusions in immediate recall. J. Exp. Psychol. 58, 17-22. doi: 10.1037/h0046671

Fabbri, M., Mencarelli, C., Adan, A., and Natale, V. (2013). Time-of-day and circadian typology on memory retrieval. Biol. Rhythm Res. 44, 125-142. doi: 10.1080/09291016.2012.656244

Farahani, F. V., Fafrowicz, M., Karwowski, W., Bohaterewicz, B., Sobczak, A. M., Ceglarek, A., et al. (2021). Identifying diurnal variability of brain connectivity 
patterns using graph theory. Brain Sci. 11:111. doi: 10.3390/brainsci110 10111

Fink, G. R., Halligan, P. W., Marshall, J. C., Frith, C. D., Frackowiak, R. S. J., and Dolan, R. J. (1996). Where in the brain does visual attention select the forest and the trees? Nature 382, 626-628. doi: 10.1038/382626a0

Frey, S., and Petrides, M. (2000). Orbitofrontal cortex: a key prefrontal region for encoding information. Proc. Natl. Acad. Sci. U. S. A. 97, 8723-8727. doi: 10.1073/pnas.140543497

Gaggioni, G., Maquet, P., Schmidt, C., Dijk, D.-J., and Vandewalle, G. (2014). Neuroimaging, cognition, light and circadian rhythms. Front. Syst. Neurosci. 8:126. doi: $10.3389 /$ fnsys.2014.00126

Garoff-Eaton, R. J., Slotnick, S. D., and Schacter, D. L. (2006). Not all false memories are created equal: the neural basis of false recognition. Cereb. Cortex 16, 1645-1652. doi: 10.1093/cercor/bhj101

Gazzaniga, M. S., Bogen, J. E., and Sperry, R. W. (1965). Observations on visual perception after disconnexion of the cerebral hemispheres in man. Brain 88 , 221-236. doi: 10.1093/brain/88.2.221

Guo, Y., Schmitz, T. W., Mur, M., Ferreira, C. S., and Anderson, M. C. (2018). A supramodal role of the basal ganglia in memory and motor inhibition: meta-analytic evidence. Neuropsychologia 108, 117-134. doi: 10. 1016/j.neuropsychologia.2017.11.033

Gutchess, A. H., and Schacter, D. L. (2012). The neural correlates of gist-based true and false recognition. NeuroImage 59, 3418-3426. doi: 10.1016/j.neuroimage. 2011.11.078

Hampson, M., Driesen, N., Roth, J. K., Gore, J. C., and Constable, R. T. (2010). Functional connectivity between task-positive and task-negative brain areas and its relation to working memory performance. Magn. Reson. Imag. 28, 1051-1057. doi: 10.1016/j.mri.2010.03.021

Jiang, X., Lv, J., Zhu, D., Zhang, T., Hu, X., Guo, L., et al. (2014). ”Integrating group-wise functional brain activities via point processes," in 2014 IEEE 11th International Symposium on Biomedical Imaging (ISBI), (Beijing: IEEE), 669-672.

Johns, M. W. (1991). A new method for measuring daytime sleepiness: the epworth sleepiness scale. Sleep 14, 540-545. doi: 10.1093/sleep/14.6.540

Karahanoğlu, F. I., and Van De Ville, D. (2015). Transient brain activity disentangles fMRI resting-state dynamics in terms of spatially and temporally overlapping networks. Nat. Commun. 6:7751. doi: 10.1038/ncomms8751

Kim, H., and Cabeza, R. (2006). Differential contributions of prefrontal, medial temporal, and sensory-perceptual regions to true and false memory formation. Cereb. Cortex 17, 2143-2150. doi: 10.1093/cercor/bhl122

Kurkela, K. A., and Dennis, N. A. (2016). Event-related fMRI studies of false memory: an Activation Likelihood Estimation meta-analysis. Neuropsychologia 81, 149-167. doi: 10.1016/j.neuropsychologia.2015.12.006

Lenth, R. V. (2021). Emmeans: Estimated Marginal Means, Aka Least-Squares Means. Available Online at: https://CRAN.R-project.org/package=emmeans (accessed October 23, 2021).

Lewandowska, K., Gagol, A., Sikora-Wachowicz, B., Marek, T., and Fafrowicz, M. (2019). Saying "yes" when you want to say "no" - pupil dilation reflects evidence accumulation in a visual working memory recognition task. Int. J. Psychophysiol. 139, 18-32. doi: 10.1016/j.ijpsycho.2019. 03.001

Lewandowska, K., Wachowicz, B., Marek, T., Oginska, H., and Fafrowicz, M. (2018). Would you say "yes" in the evening? Time-of-day effect on response bias in four types of working memory recognition tasks. Chronobiol. Int. 35, 80-89. doi: 10.1080/07420528.2017.1386666

Li, W., Li, Y., Hu, C., Chen, X., and Dai, H. (2014). Point process analysis in brain networks of patients with diabetes. Neurocomputing 145, 182-189. doi: 10.1016/j.neucom.2014.05.045

Libby, L. A., Hannula, D. E., and Ranganath, C. (2014). Medial temporal lobe coding of item and spatial information during relational binding in working memory. J. Neurosci. 34, 14233-14242. doi: 10.1523/jneurosci.0655-14. 2014

Liu, X., and Duyn, J. H. (2013). Time-varying functional network information extracted from brief instances of spontaneous brain activity. Proc. Natl. Acad. Sci. U. S. A. 110, 4392-4397. doi: 10.1073/pnas.1216856110

Liu, X., Chang, C., and Duyn, J. (2013). Decomposition of spontaneous brain activity into distinct fMRI co-activation patterns. Front. Syst. Neurosci. 7:101. doi: $10.3389 /$ fnsys. 2013.00101
Loftus, E. F. (1979). The malleability of human memory. Am. Sci. 67, 312-320.

Marek, T., Fafrowicz, M., Golonka, K., Mojsa-Kaja, J., Oginska, H., Tucholska, K., et al. (2010). Diurnal patterns of activity of the orienting and executive attention neuronal networks in subjects performing a stroop-like task: a functional magnetic resonance imaging study. Chronobiol. Int. 27, 945-958. doi: 10.3109/ 07420528.2010.489400

Martinez, A., Moses, P., Frank, L., Buxton, R., Wong, E., and Stiles, J. (1997). Hemispheric asymmetries in global and local processing. NeuroReport 8, 16851689. doi: 10.1097/00001756-199705060-00025

McNab, F., and Klingberg, T. (2008). Prefrontal cortex and basal ganglia control access to working memory. Nat. Neurosci. 11, 103-107. doi: 10.1038/nn2024

Miller, E. K. (2000). The prefontral cortex and cognitive control. Nat. Rev. Neurosci. 1, 59-65. doi: 10.1038/35036228

Milner, B. (1971). Interhemispheric differences in the localization of psychological processes in man. Brit. Med. Bull. 27, 272-277. doi: 10.1093/oxfordjournals. bmb.a070866

Mitra, A., Snyder, A. Z., Hacker, C. D., and Raichle, M. E. (2014). Lag structure in resting-state fMRI. J. Neurophysiol. 111, 2374-2391. doi: 10.1152/jn.00804. 2013

O’Doherty, J. P. (2007). Lights, camembert, action! the role of human orbitofrontal cortex in encoding stimuli, rewards, and choices. Ann. N. Y. Acad. Sci. 1121, 254-272. doi: 10.1196/annals.1401.036

Oginska, H., Mojsa-Kaja, J., and Mairesse, O. (2017). Chronotype description: in search of a solid subjective amplitude scale. Chronobiol. Int. 34, 1388-1400. doi: 10.1080/07420528.2017.1372469

Olson, I. R., Page, K., Moore, K. S., Chatterjee, A., and Verfaellie, M. (2006). Working memory for conjunctions relies on the medial temporal lobe. J. Neurosci. 26, 4596-4601. doi: 10.1523/jneurosci.1923-05. 2006

Petridou, N., Gaudes, C. C., Dryden, I. L., Francis, S. T., and Gowland, P. A. (2013). Periods of rest in $\mathrm{fMRI}$ contain individual spontaneous events which are related to slowly fluctuating spontaneous activity. Hum. Brain Mapp. 34, 1319-1329. doi: $10.1002 / \mathrm{hbm} .21513$

R Core Team (2021). R: A Language and Environment for Statistical Computing. Vienna: R Foundation for Statistical Computing.

Ratcliff, R. (1978). A theory of memory retrieval. Psychol. Rev. 85, 59-108. doi: 10.1037/0033-295x.85.2.59

Ratcliff, R., and McKoon, G. (2008). The diffusion decision model: theory and data for two-choice decision tasks. Neural Comput. 20, 873-922. doi: 10.1162/neco. 2008.12-06-420

Raut, R. V., Snyder, A. Z., Mitra, A., Yellin, D., Fujii, N., Malach, R., et al. (2021). Global waves synchronize the brain's functional systems with fluctuating arousal. Sci. Adv. 7:eabf2709. doi: 10.1126/sciadv.abf2709

Roediger, H. L., and McDermott, K. B. (1995). Creating false memories: remembering words not presented in lists. J. Exp. Psychol. Learn. Mem. Cogn. 21, 803-814. doi: 10.1037/0278-7393.21.4.803

Rolls, E. T. (2019). The cingulate cortex and limbic systems for emotion, action, and memory. Brain Struct. Funct. 224, 3001-3018. doi: 10.1007/s00429-019-0 1945-2

Schacter, D. L., Norman, K. A., and Koutstaal, W. (1998). The cognitive neuroscience of constructive memory. Ann. Rev. Psychol. 49, 289-318. doi: 10.1146/annurev.psych.49.1.289

Schmidt, C., Collette, F., Cajochen, C., and Peigneux, P. (2007). A time to think: circadian rhythms in human cognition. Cogn. Neuropsychol. 24, 755-789. doi: 10.1080/02643290701754158

Schmidt, C., Collette, F., Reichert, C. F., Maire, M., Vandewalle, G., Peigneux, P., et al. (2015). Pushing the limits: chronotype and time of day modulate working memory-dependent cerebral activity. Front. Neurol. 6:199. doi: 10.3389/fneur. 2015.00199

Schmidt, C., Peigneux, P., Leclercq, Y., Sterpenich, V., Vandewalle, G., Phillips, C., et al. (2012). Circadian preference modulates the neural substrate of conflict processing across the day. PLoS One 7:e29658. doi: 10.1371/journal.pone. 0029658

Sieveritz, B., García-Muñoz, M., and Arbuthnott, G. W. (2019). Thalamic afferents to prefrontal cortices from ventral motor nuclei in decision making. Eur. J. Neurosci. 49, 646-657. doi: 10.1111/ejn.14215

Sikora-Wachowicz, B., Keresztes, A., Werkle-Bergner, M., Lewandowska, K., Marek, T., and Fafrowicz, M. (2021). False recognitions in short-term memory: 
age-differences in neural activity. Brain Cogn. 151:105728. doi: 10.1016/j.bandc. 2021.105728

Sikora-Wachowicz, B., Lewandowska, K., Keresztes, A., Werkle-Bergner, M., Marek, T., and Fafrowicz, M. (2019). False recognition in short-term memory age-differences in confidence. Front. Psychol. 10:2785. doi: 10.3389/fpsyg.2019. 02785

Sirotin, Y. B., and Das, A. (2009). Anticipatory haemodynamic signals in sensory cortex not predicted by local neuronal activity. Nature 457, 475-479. doi: 10 . 1038/nature07664

Slotnick, S. D., and Schacter, D. L. (2004). A sensory signature that distinguishes true from false memories. Nat. Neurosci. 7, 664-672. doi: 10.1038/nn1252

StatSoft, Inc (2012). Electronic Statistics Textbook. Tulsa: StatSoft.

Steiner, A. P., and Redish, A. D. (2012). The road not taken: neural correlates of decision making in orbitofrontal cortex. Front. Neurosci. 6:131. doi: 10.3389/ fnins.2012.00131

Tagliazucchi, E., Balenzuela, P., Fraiman, D., and Chialvo, D. R. (2012). Criticality in large-scale brain fMRI dynamics unveiled by a novel point process analysis. Front. Physiol. 3:15. doi: 10.3389/fphys.2012.00015

Tagliazucchi, E., Balenzuela, P., Fraiman, D., Montoya, P., and Chialvo, D. R. (2011). Spontaneous BOLD event triggered averages for estimating functional connectivity at resting state. Neurosci. Lett. 488, 158-163. doi: 10.1016/j.neulet. 2010.11.020

Tandoc, M. C., Bayda, M., Poskanzer, C., Cho, E., Cox, R., Stickgold, R., et al. (2021). Examining the effects of time of day and sleep on generalization. PLoS One 16:e0255423. doi: 10.1371/journal.pone.0255423

Tzourio-Mazoyer, N., Landeau, B., Papathanassiou, D., Crivello, F., Etard, O., Delcroix, N., et al. (2002). Automated anatomical labeling of activations in SPM using a macroscopic anatomical parcellation of the MNI MRI single-subject brain. NeuroImage 15, 273-289. doi: 10.1006/nimg.2001.0978

Weissman, D. H., and Woldorff, M. G. (2004). Hemispheric Asymmetries for Different Components of Global/Local Attention Occur in Distinct Temporoparietal Loci. Cerebral. Cortex 15, 870-876. doi: 10.1093/cercor/bhh18

Welniarz, Q., Gallea, C., Lamy, J., Méneret, A., Popa, T., Valabregue, R., et al. (2019). The supplementary motor area modulates interhemispheric interactions during movement preparation. Hum. Brain Mapp. 40, 2125-2142. doi: 10.1002/hbm. 24512

Wu, G.-R., Liao, W., Stramaglia, S., Ding, J.-R., Chen, H., and Marinazzo, D. (2013). A blind deconvolution approach to recover effective connectivity brain networks from resting state fMRI data. Med. Image Anal. 17, 365-374. doi: 10.1016/j.media.2013.01.003

Yan, C.-G., Wang, X.-D., Zuo, X.-N., and Zang, Y.-F. (2016). DPABI: data processing \& analysis for (Resting-State) brain imaging. Neuroinformatics 14, 339-351. doi: 10.1007/s12021-016-9299-4

Yonelinas, A. P. (2013). The hippocampus supports high-resolution binding in the service of perception, working memory and long-term memory. Behav. Brain Res. 254, 34-44. doi: 10.1016/j.bbr.2013.05.030

Zuanazzi, A., and Cattaneo, L. (2017). The right hemisphere is independent from the left hemisphere in allocating visuospatial attention. Neuropsychologia 102, 197-205. doi: 10.1016/j.neuropsychologia.2017.06.005

Conflict of Interest: The authors declare that the research was conducted in the absence of any commercial or financial relationships that could be construed as a potential conflict of interest.

Publisher's Note: All claims expressed in this article are solely those of the authors and do not necessarily represent those of their affiliated organizations, or those of the publisher, the editors and the reviewers. Any product that may be evaluated in this article, or claim that may be made by its manufacturer, is not guaranteed or endorsed by the publisher.

Copyright (C) 2021 Ceglarek, Ochab, Cifre, Fafrowicz, Sikora-Wachowicz, Lewandowska, Bohaterewicz, Marek and Chialvo. This is an open-access article distributed under the terms of the Creative Commons Attribution License (CC BY). The use, distribution or reproduction in other forums is permitted, provided the original author(s) and the copyright owner(s) are credited and that the original publication in this journal is cited, in accordance with accepted academic practice. No use, distribution or reproduction is permitted which does not comply with these terms. 